\title{
Parakardiales Infiltrat oder türkischer Säbel?
}

\author{
M. Riedel ${ }^{1}$ \\ S. Martinoff ${ }^{2}$ \\ J. Hausleiter ${ }^{1}$ \\ V. Bohanes $^{3}$ \\ C. Engelke ${ }^{4}$
}

\section{Paracardiac Infiltrate or Turkish Sword?}

\section{Zusammenfassung}

Der Artikel berichtet über vier Fälle des Scimitar-Syndroms mit typischen radiologischen Befunden. Die Kasuistiken zeigen, dass beim Vorliegen eines gekrümmten Gefäßschattens oder einer bogig begrenzten Verschattung rechts parakardial in der posterio-anterioren Thoraxaufnahme auch an eine anomale Lungenvenenmündung gedacht werden sollte. Diese Anomalie kann bis ins Erwachsenenalter asymptomatisch bleiben.

\section{Abstract}

Four cases of the scimitar syndrome with typical radiologic findings are described. The cases demonstrate that when a curved density along the right paracardial border that descends toward the diaphragm is visible on the posteroanterior chest radiograph, the possibility of an anomalous connection of the pulmonary vein with the inferior vena cava should be considered. The anomaly can remain asymptomatic until adult life.
Eine von der Norm abweichende Thoraxaufnahme stellt einen häufigen Grund für eine Überweisung des Patienten zum Kardiologen oder Pneumologen dar. Eine parakardiale Verschattung beim asymptomatischen oder nur leicht dyspnoischen Patienten wird zunächst einem entzündlichen Infiltrat zugeschrieben. Nach einer erfolglosen antibiotischen Therapie folgen dann weitere diagnostische Schritte zum Ausschluss eines Bronchialkarzinoms als Ursache der vermeintlichen Atelektase. Die folgende Fallbeispiele sollen darauf hinweisen, dass es sich bei Verschattungen im rechten Unterlappen auch um die erste Manifestation einer angeborenen Gefäßanomalie handeln kann.

\section{Fallbeispiele}

\section{Patient Nr. 1}

Ein asymptomatischer 66-jähriger Mann kam zur Untersuchung, da in der Röntgenaufnahme des Thorax ein Infiltrat befundet wurde, das unter antibiotischer Therapie keine Resorptionstendenz aufwies. Die Thoraxaufnahme zeigte prominente Zentraläste der Pulmonalarterie (PA) mit normalem Kaliber in der Peripherie sowie eine typische scimitarförmige Verschattung in der rechten Lunge (Abb.1, Pfeile). Bei der Herzauskultation wurde ein leises Ejektionssystolikum über der Pulmonalklappe und ein leises Strömungs-Mesodiastolikum über dem unteren Sternum gehört, welches den Verdacht auf einen Links-rechts-Shunt erweckte. Der zweite Herzton wies jedoch eine normale respirationsabhängige Spaltung auf. Echokardiographisch wurde ein dilatierter rechter Ventrikel (RV) mit normaler Kinetik und ein sys-

Institutsangaben

${ }^{1}$ Klinik für Herz- und Kreislauferkrankungen, Deutsches Herzzentrum, Technische Universität München

${ }^{2}$ Institut für Radiologie und Nuklearmedizin, Deutsches Herzzentrum, Technische Universität München

${ }^{3}$ Bezirks-Krankenhaus, St. Johann in Tirol, Österreich

${ }^{4}$ Institut für Radiologie, Klinikum rechts der Isar, Technische Universität München

Korrespondenzadiresse

Doz. Dr. med. M. Riedel · Deutsches Herzzentrum und I. Medizinische Klinik · Technische Universität München Lazarettstraße 36·80636 München·E-mail: m.riedel@dhm.mhn.de

Eingereicht: 5. Oktober 2002 · Nach Überarbeitung angenommen: 9. Dezember 2002

Bibliografie

Pneumologie 2003; 57: 78-82 @ Georg Thieme Verlag Stuttgart · New York · ISSN 0934-8387 


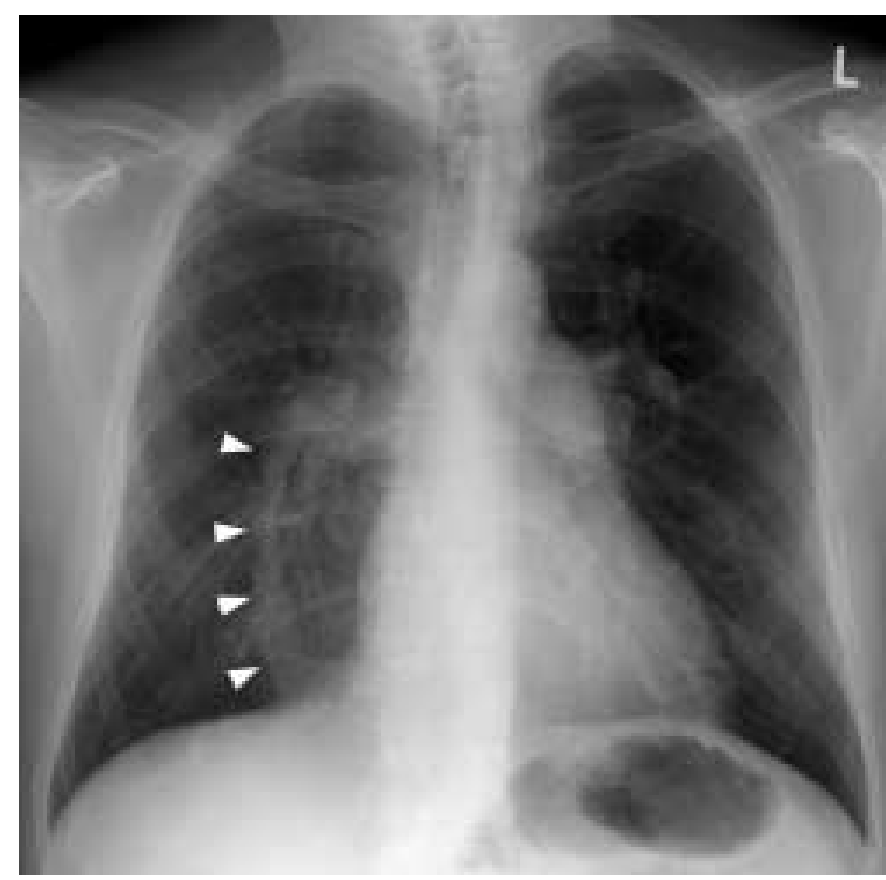

Abb. 1 Röntgenaufnahme des Thorax von Patient Nr. 1 mit einer scimitarförmigen Verschattung im rechten Unterfeld (Pfeile).

tolischer Gradient von $25 \mathrm{~mm} \mathrm{Hg}$ zwischen dem rechten Ventrikel und dem rechten Vorhof nachgewiesen; ein Vorhofseptumdefekt konnte ausgeschlossen werden. Der linke Ventrikel und alle Klappen waren unauffällig. Die ultraschnelle, EKG-getriggerte 16-Zeilen-Multislice-Computertomographie (CT, Somatom Sensation Cardiac) mit Kontrastmittel zeigte eine dilatierte PA (Stammdurchmesser $36 \mathrm{~mm}$ ), einen dilatierten RV, eine normale arterielle Versorgung beider Lungen und eine Scimitarvene (SV) mit Mündung in die untere Hohlvene (IVC) (Abb.2a). In der Frontalebene (Abb. 2b) ist die SV in ihrem ganzen Verlauf bis zur IVC dargestellt; die Abbildung zeigt auch die dilatierte PA. Die dreidimensionale Rekonstruktion der CT-Bilder (Abb. 2c u. d) wies eindeutig nach, dass nahezu die gesamte venöse Rückkehr aus der rechten Lunge über die anomale Scimitarvene in die IVC knapp über dem Zwerchfell geleitet wurde. Die linksseitigen Lungenvenen mündeten normal in den linken Vorhof (LA) hinein (Abb. 2d). Die Katheteruntersuchung bestätigte die anomale venöse Rückkehr über die Scimitarvene mit einem Linksrechts-Shunt von $48 \%$. Angesichts der Symptomlosigkeit, der Abwesenheit anderer kardiovaskulärer Anomalien oder einer pulmonalen Hypertonie wurde von einer Korrekturoperation abgesehen.

\section{Patientin Nr. 2}

Bei dieser Patientin wurde am 8. Lebenstag aufgrund pathologischer Atemgeräusche bei der Auskultation der Verdacht auf eine Pneumonie in den Raum gestellt. Wegen der - trotz antibiotischer Therapie - ausbleibenden radiologischen Besserung wurde in der sechsten Lebenswoche eine Herzkatheteruntersuchung durchgeführt, die einen kleinen Vorhofseptumdefekt (vom Secundum-Typ), einen hypoplastischen rechten Pulmonalisast, eine arterielle Versorgung des rechten Lungenunterlappens aus der Aorta abdominalis (ähnlich der Lungensequestration) und eine anomale Mündung der rechten unteren Lungenvene in die untere Hohlvene knapp vor deren Mündung in den rechten Vorhof nachweisen konnte. Das Verhältnis des pulmonalen zum systemischen Flusses (Qp/Qs) betrug 2,03:1, der Pulmonalisdruck war normal. Es wurde beschlossen, in einem Zeitabstand den Vorhofseptumdefekt zu verschließen und eine Ligatur der aberranten Arterie zum rechten Unterlappen durchzuführen. Im weiteren Verlauf gedieh jedoch das Kind sehr gut und wiederholte echokardiographische Untersuchungen zeigten einen normalen Pulmonalisdruck mit normal großem rechten Ventrikel. Bei der letzten ambulanten Kontrolle fand sich in der Röntgenaufnahme des Thorax (Abb. 3 links) eine leichte kardiale Dextroposition, eine leichte Hypoplasie der rechten Lunge, das typische Scimitarzeichen und ein prominenter, zur Peripherie hin schmäler werdender linker Pulmonalisast sowie leichte Rezirkulationszeichen. Ein Katheter-Vorhofseptumverschluss oder eine komplette chirurgische Korrektur wurde von der mittlerweile bereits 24-jährigen asymptomatischen Frau abgelehnt.

\section{Patientin Nr. 3}

Bei einer 20-jährigen asymptomatischen Frau ohne eine relevante Vorgeschichte wurde bei einer Routineröntgenuntersuchung (Abb. 3 rechts) eine Dextroposition des Herzens, eine leichte Hypoplasie der rechten Lunge, ein angedeutetes Scimitarzeichen mit sonst normaler Pulmonalisverästelung und leichten Rezirkulationszeichen festgestellt. Bei der Katheteruntersuchung wurde eine Scimitarvene als einzige Ursache eines Links-rechts-Shunts von 55\% nachgewiesen; der Pulmonalisdruck war normal. Die Patientin wurde in der Folge operiert, auf eigenen Wunsch unter Anwendung eines minimalinvasiven Zugangs aus einer Thorakotomie rechts anterolateral. Nach dem Anschluss des Extrakorporalkreislaufes aus diesem Zugang wurde die Mündung der anomalen Lungenvene in die untere Hohlvene auf Zwerchfellebene identifiziert und mittels eines Tunnels aus einer aufgeschnittenen Goretex-Prothese mit einem neu erschaffenen Vorhofseptumdefekt verbunden. Am dritten postoperativen Tag kam es zu einer rasch progredienten Verschattung der rechten Lunge mit Zeichen der rechtsventrikulären Überlastung; echokardiographisch konnte (im Unterschied zur unmittelbaren postoperativ durchgeführten Untersuchung) kein Fluss im Tunnel nachgewiesen werden. Bei der Herzkatheteruntersuchung konnten nach der Kontrastverabreichung in den rechten Pulmonalisast alle Arterienäste bis in die Peripherie dargestellt werden, jedoch keine kapillare oder venöse Phase. Das Kontrastmittel bewegte sich in diesem Bereich hin und her, bis es allmählich in den linken Pulmonalisast abfloss. Nach der Injektion des Kontrastmittels in den linken Vorhof konnte das Tunnelostium, aber keine Verbindung zum rechten Vorhof oder zu einer Lungenvene dargestellt werden; auch mittels selektiven Katheters gelang es nicht, in den Tunnel einzudringen. Bei der unmittelbar folgenden Reoperation, diesmal aus medialer Sternotomie, wurde - wie bereits aufgrund der Angiographie angenommen - ein thrombotischer Tunnelverschluss festgestellt. Der Tunnel wurde reseziert, die Scimitarvene präpariert und mittels einer breiten Anastomose mit der lateralen rechtsatrialen Wand verbunden; von hier aus wurde über einen neuen Goretex-Tunnel die Verbindung mit dem linken Vorhof hergestellt. Der weitere Verlauf war komplikationslos, die Patientin wurde 3 Monate lang oral antikoaguliert und ist ein Jahr nach der Operation beschwerdefrei. 


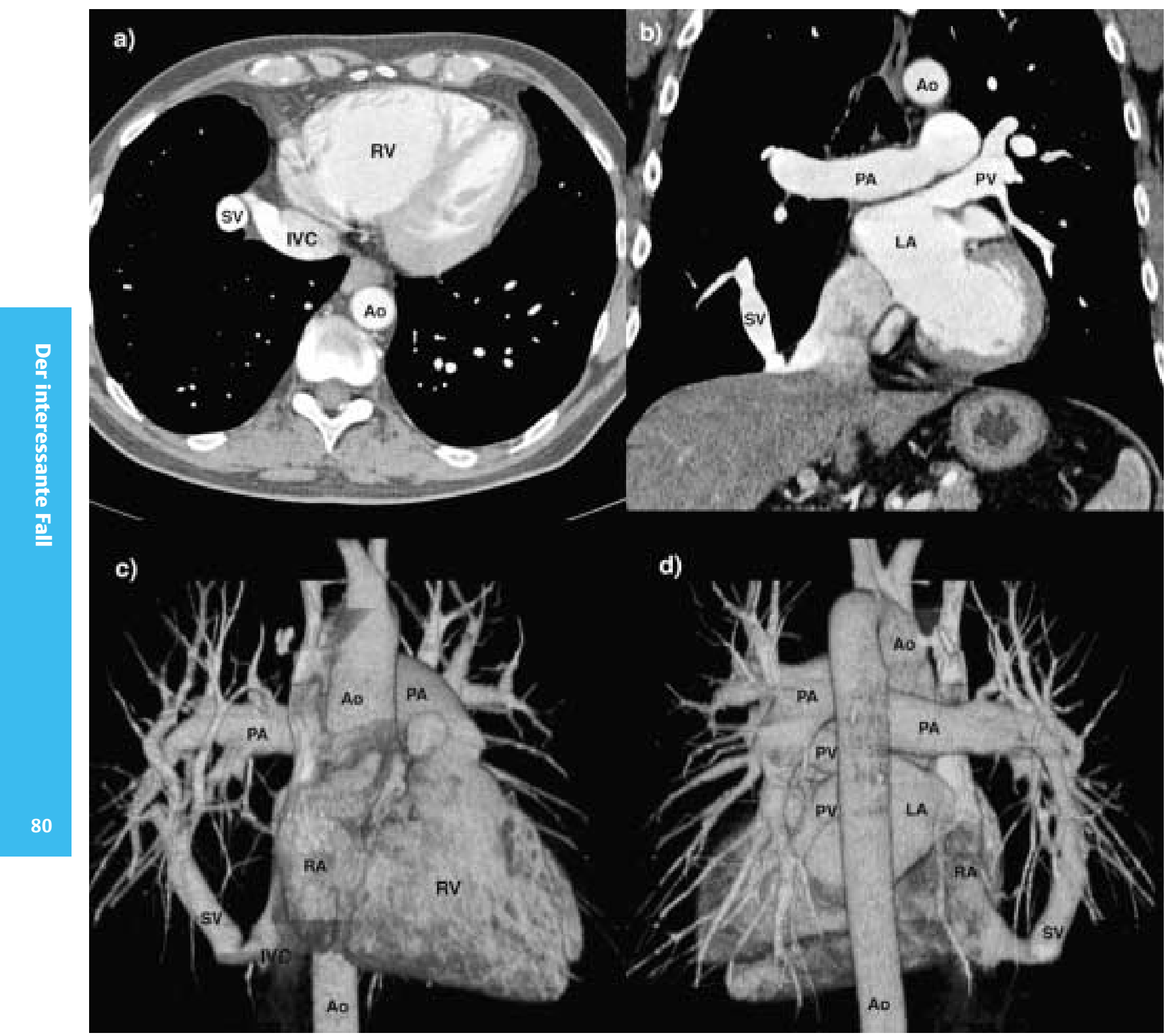

Abb. 2 Multislice-CT von Patient Nr. 1. a) Horizontalebene auf Höhe der Zwerchfellkuppel, b) Frontalebene, c) und d) dreidimensionale Rekonstruktion von ventral und dorsal betrachtet. PA - Lungenarterie; PV - Lungenvene; SV - anomale pulmonale Scimitarvene; RV - rechter Ventrikel; RA - rechter Vorhof; LA - linker Vorhof; IVC - untere Hohlvene; Ao - Aorta.

\section{Patient Nr. 4}

Bei einem 45-jährigen asymptomatischen Mann wurde bei einer Allgemeinuntersuchung im Rahmen einer Blutspende eine Hypoplasie der rechten Lunge mit Dextroposition des Herzens und einer parakardialen scimitarförmigen Verschattung festgestellt. Die digitale Subtraktionsangiographie mit Kontrastmittelinjektion in die linke PA (Abb.4) zeigte eindeutig die Verschattungsursache. Von einer Korrekturoperation wurde bei diesem Patienten abgesehen.

\section{Diskussion}

Das Scimitarsyndrom stellt eine Sonderform der partialen anomalen Lungenvenenmündung dar. Eine oder mehrere Venen der rechten Lunge münden über eine Sammelvene in die untere Hohlvene oder die Portalvene hinein. Dies führt in der Regel zu einem charakteristischen radiologischen Bild einer breiten gewölbeartigen Verschattung entlang des rechten Herzrandes bis zum Zwerchfell (Abb.1 u. 4). Dieser Schatten erinnert an den türkischen Säbel - Scimitar - und daher kommt auch die Bezeichnung. Das Scimitarzeichen im posterior-anteriorem Thoraxröntgenbild ist für das Scimitarsyndrom jedoch nicht spezifisch: außer der inkompletten anomalen Lungenvenenmündung (d.h. dem eigentlichen Scimitarsyndrom) kann es auch die Folge von 

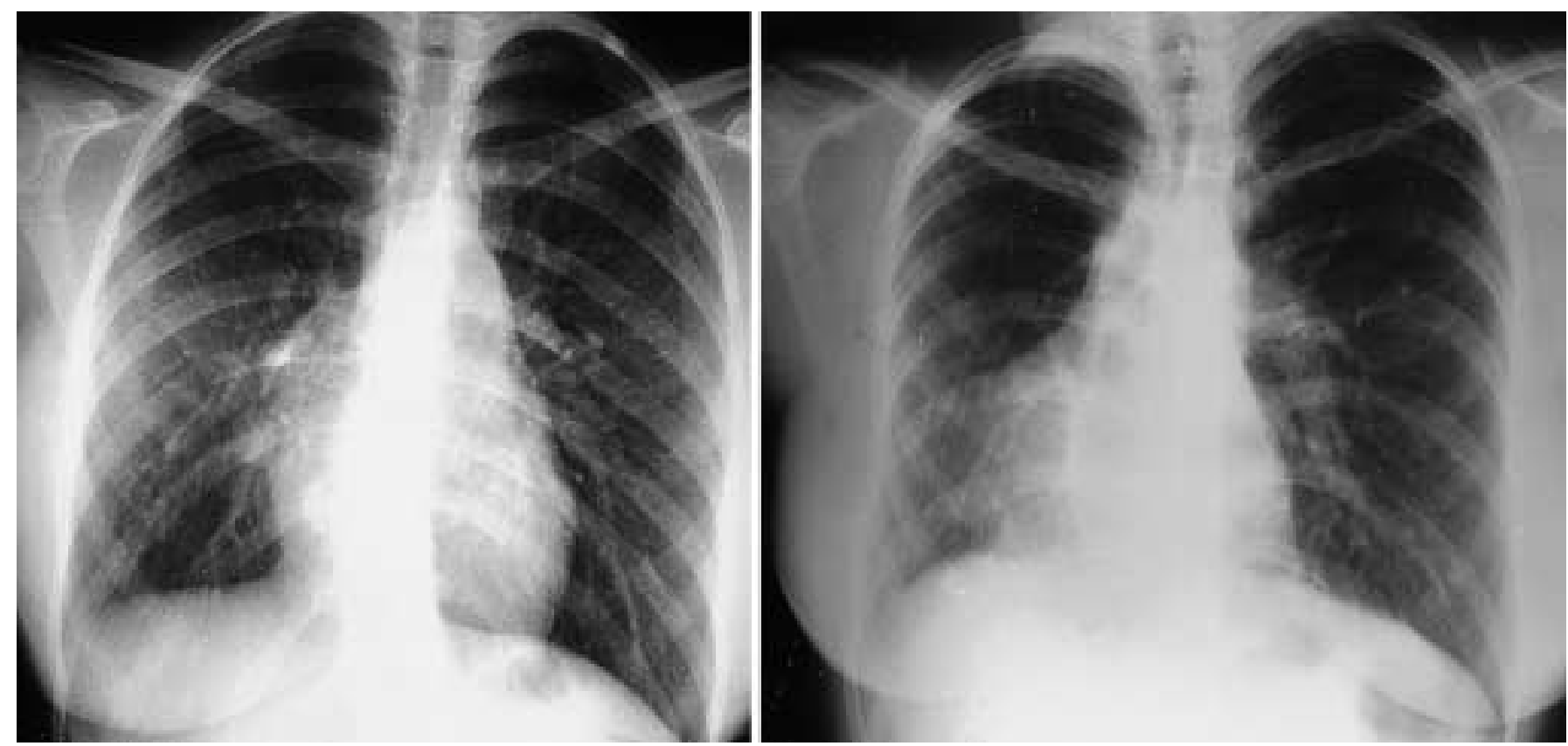

Abb. 3 Röntgenaufnahme des Thorax von Patientin Nr. 2 (links) und Nr. 3 (rechts). In beiden Fällen eine Dextroposition des Herzens (welche die Scimitarvene teilweise überdeckt) und eine leichte Hypoplasie der rechten Lunge.

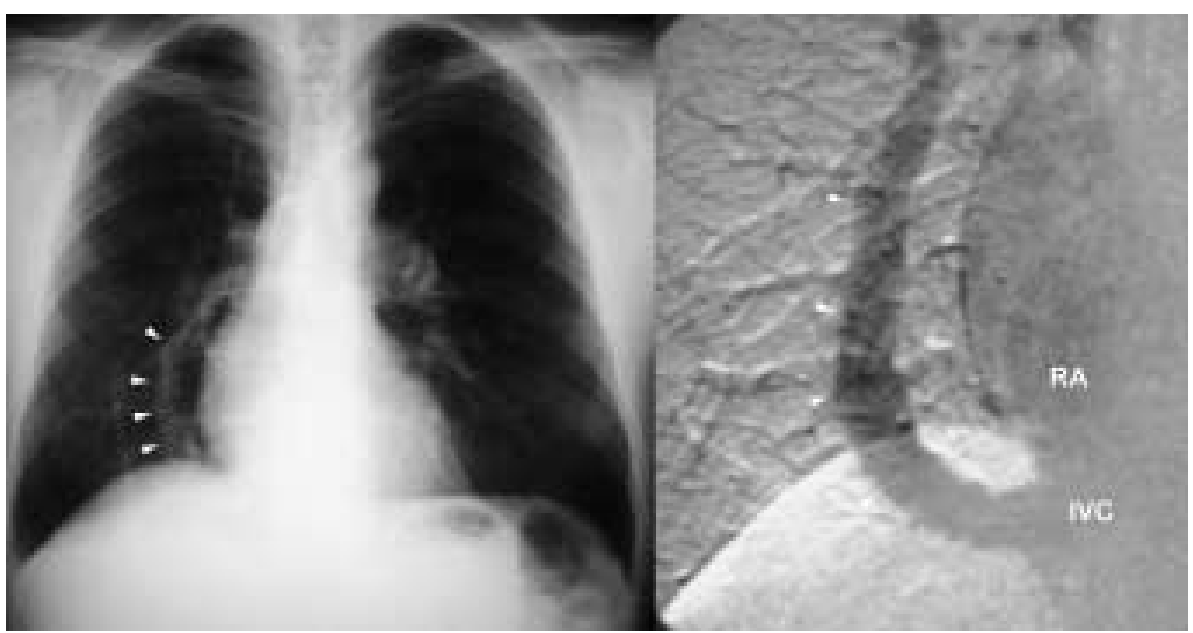

Abb. 4 Röntgenaufnahme des Thorax und DSA mit Kontrastmittelinjektion in die linke PA beim Patienten Nr. 4. DSA der venösen Phase zeigt die anomale Mündung der Scimitarvene in die untere Hohlvene (IVC) knapp unterhalb des Zwerchfells. RA - rechter Vorhof.

pulmonalen arteriovenösen Malformationen, einer Lungenvarix, einer pulmonalen Meandervene oder einer anomalen intrapulmonalen Verbindung von normal mündenden Lungenvenen sein. Bei kardialer Dextroposition (Abb.3) muss das Scimitarzeichen jedoch nicht typisch ausfallen, so dass weitere bildgebende Verfahren nötig werden. Die Scimitarvene drainiert in der Regel die gesamte rechte Lunge, bei einigen Patienten allerdings nur den Unterlappen, ggf. den Mittellappen (in diesen Fällen werden die oberen Bereiche normal in den linken Vorhof drainiert).

Angesichts signifikanter Unterschiede in der Morbidität und Mortalität wird zwischen zwei Scimitar-Syndrom-Typen unterschieden: dem infantilen Typ (diagnostiziert im Säuglingsalter) und dem „Erwachsenentyp“ (diagnostiziert nach dem ersten Lebensjahr) $[1,2]$. Bei der infantilen Form liegt häufig auch eine anomale arterielle Versorgung der rechten Lunge direkt aus der Aorta vor (wie bei unserer Patientin Nr. 2), die rechte Lunge ist oft hypoplastisch mit Anomalien des Bronchialbaumes und gelegentlichen Sequestrierungen [2,3]. Das Herz ist nach rechts disloziert, das Vorhofseptum jedoch zumeist normal. Häufig liegt auch eine pulmonale Hypertonie vor, die auf mehrere Faktoren zurückgeführt wird, z.B. einen Links-rechts-Shunt bei anomaler Lungenvenenmündung oder assoziierten Defekten, eine Versorgung der Lunge über eine Systemarterie, eine Lungenhypoplasie mit folgender Restriktion des Lungengefäßbettes, eine pulmonalvenöse Obstruktion oder anhaltende pulmonale Hypertonie bei Neugeborenen [4]. Die Kinder leiden an häufigen respiratorischen Infekten.

Bei der sog. „Erwachsenenform“ (die per Definitionen sowohl Kinder als auch Erwachsene beinhaltet) liegt oft ein Vorhofseptumdefekt vor. Viele Erwachsene sind asymptomatisch und die Anomalie wird zufällig bei einer Röntgenuntersuchung entdeckt $[1,5]$; symptomatische Patienten sind überwiegend Kinder mit einem großen Links-rechts-Shunt und assoziierten Herzfehlern. 
Der Volumendatensatz und die dreidimensionale Bildrekonstruktion der Multislice-CT-Angiographie weisen eindeutig die anomale Scimitarvene und deren anomale Mündung nach (Abb. 2). Die Magnetresonanztomographie wird wahrscheinlich eine Alternative werden, da sie nichtinvasiv und strahlenbelastungsfrei ist und ähnlich wie die CT ein breites Betrachtungsfeld in multiplen Ebenen bietet; zudem ermöglicht sie eine Berechnung des Links-rechts-Shunts durch die direkte Blutflussmessung in der Aorta, dem Pulmonalisstamm und der aberranten Lungenvene. Allerdings bietet die CT-Angiographie zusätzlich Information über die Lungenanatomie (was in der Differenzialdiagnostik der Lungesequestration von besonderer Bedeutung ist) und sie ist somit zur Zeit der Magnetresonanz-Angiographie überlegen. Die Magnetresonanz-Angiographie ist aufgrund der fehlenden Strahlenexposition für die Beurteilung pädiatrischer Patienten im Allgemeinen vorzuziehen. Die Herzkatheteruntersuchung mit Lungenangiographie (klassisch oder DSA) in der venösen Phase ist nur bei einer Operationserwägung unumgänglich, vor allem zum Ausschluss assoziierter angeborener Anomalien.

Im Erwachsenenalter ist die Operation eines unkomplizierten Scimitar-Syndroms mit größeren Links-rechts-Shunt nur bei Entwicklung einer pulmonalen Hypertonie oder rechtsventrikulären Dilatation notwendig; beide können unschwer bei echokardiographischen Verlaufskontrollen festgestellt werden. Die Korrektur besteht in der Anbindung der anomalen Vene mittels einer direkten Anastomose an den linken Vorhof oder mittels eines Tunnels zwischen der Venenmündung und einem neu geschaffenen Vorhofseptumdefekt (wie bei unserer Patientin Nr. 3 ) oder mittels eines Perikardpatches, der von der unteren Hohlvene ausgehend an der Wand des rechten Vorhofs entlang über das offene Foramen ovale in den linken Vorhof mündet [4]. Diese
Operation kann technisch schwierig sein; ein gelegentlicher postoperativer thrombotischer (trotz Antikoagulation) oder fibrotischer Verschluss der Vene wird u. a. einem unzureichenden Blutfluss über die Lungenarterie zugeschrieben [3]. Die Erfahrung mit unserer Patientin Nr. 3 untermauert die überwiegende Meinung von Kardiologen, nach der der derzeitige Modetrend zur minimalinvasiven Chirurgie bei technisch schwierigen Operationen trotz guten kosmetischen Ergebnissen nicht immer die optimale Methode sein muss.

Die vorliegenden Fallbeispiele demonstrieren, dass beim Befund einer gekrümmten Gefäßverschattung rechts parakardial auf der postero-anterioren Aufnahme an eine inkomplette Lungenvenenmündung gedacht werden sollte. Diese Anomalie kann bis ins Erwachsenenalter asymptomatisch bleiben.

\section{Literatur}

${ }^{1}$ Dupuis C, Charaf LAC, Breviere GM et al. The "adult" form of the scimitar syndrome. Am J Cardiol 1992; 70: $502-507$

2 Dupuis C, Charaf LA, Breviere GM et al. "Infantile" form of the scimitar syndrome with pulmonary hypertension. Am J Cardiol 1993; 71: $1326-1330$

${ }^{3}$ Schramel FM, Westermann CJ, Knaepen PJ et al. The scimitar syndrome: clinical spectrum and surgical treatment. Eur Respir J 1995; 8: $196-201$

${ }^{4}$ Najm HK, Williams WG, Coles JG et al. Scimitar syndrome: twenty years' experience and results of repair. J Thorac Cardiovasc Surg 1996; 112: $1161-1168$

${ }^{5}$ Idris MT. Diagnostic aid of transesophageal echocardiography in an adult case of scimitar syndrome: confirmation of the findings at surgery and review of the literature. J Am Soc Echocardiogr 1998; 11: $387-392$ 